\title{
Mecanoreceptores da mucosa palatina de avestruz (Struthio camelus): estudo ao microscópio de luz ${ }^{1}$
}

\author{
Juliana P. Guimarães ${ }^{2 *}$, Renata B. Mari², Maria Angélica Miglino², Francisco J. \\ Hernandez-Blasquez ${ }^{2}$ e Ii-sei Watanabe ${ }^{2}$
}

\begin{abstract}
Guimarães J.P., Mari R.B., Miglino M.A., Hernandez-Blasquez F.J. \& Watanabe I. 2007. [Mechanoreceptors of the palatine mucosa of ostrich (Struthio camelus): light microscope study.] Mecanoreceptores da mucosa palatina de avestruz (Struthio camelus): estudo ao microscópio de luz. Pesquisa Veterinária Brasileira 27(12):491-494. Departamento de Cirurgia, Setor de Anatomia, Faculdade de Medicina Veterinária e Zootecnia, Universidade de São Paulo, Av. Prof. Dr. Orlando Marques de Paiva 87, São Paulo, SP 05508-270, Brazil. E-mail: juvet@usp.br

Herbst corpuscles of the palatine mucosa of ostrich were studied by light microscopy. The corpuscles are composed of an outer core, inner core and central nerve terminal. The outer core presents numerous lamellae, while the inner core shows compact structure of cytoplasm sheets. The corpuscles are elongate or oval in shape and are surrounded by bundles of collagen fibers. Each lamella is composed of a dense network of thick fibrils. The terminal axons are located along the axis and form a bulb terminal. The fibers of external core stained by Picrosirius and examined by polarized light microscopy revealed to be green in color like type I collagen fibers, and at the periphery is a large amount of collagen type III. The corpuscles are surrounded by flat cells and dense collagen fibers at the periphery.
\end{abstract}

INDEX TERMS: Mechanoreceptors, palatine mucosa, ostrich, light microscope.

RESUMO.- Foram estudados corpúsculos de Herbst da mucosa palatina de avestruz em nível de microscopia de luz. Os corpúsculos compõem-se de uma cápsula externa, cápsula interna e axônio central. A cápsula externa apresentou numerosas lamelas, enquanto que a cápsula interna mostrou estrutura de folhas compactas. Os corpúsculos apresentaram formato ovalado ou circular e circundado por espessos feixes de fibras colágenas. Cada lamela estava composta de uma densa rede de fibras espessas. Os axônios terminais estavam situados ao longo do eixo, terminando em um bulbo terminal. As fibras da cápsula externa, coradas por Picrosirius e examinadas no microscópio óptico sob luz polarizada, revelou a presença de fibras colágenas do tipo I em verde e na região periférica observou-se grande quantidade de fibras colágenas do tipo III. Os corpúsculos apresentaram-se envoltos por células planas e envoltos por fibras colágenas.

\footnotetext{
${ }^{1}$ Recebido em 19 de junho de 2007.

Aceito para publicação em 3 de dezembro de 2007.

2 Departamento de Cirurgia, Setor de Anatomia, Faculdade de Medicina Veterinária e Zootecnia, Universidade de São Paulo, Av. Prof. Dr. Orlando Marques de Paiva 87, Cidade Universitária, São Paulo, SP 05508-270, Brasil. *Autor para correspondência: juvet@usp.br
}

TERMOS DE INDEXAÇÃO: Mecanorreceptores, mucosa palatina, avestruz, microscopia de luz.

\section{INTRODUÇÃO}

O tecido conjuntivo da mucosa oral contém terminações nervosas organizadas encapsuladas simples ou múltiplas com axônio terminal central e sistema de lamelas dos núcleos interno e externo. Os corpúsculos lamelares são formados por um axônio central envolto por citoplasma de células lamelares de disposição simétrica no núcleo interno como relatado por Pease \& Quilliam (1957), Cauna \& Mannan (1958), Andres (1969), Spencer \& Schaumburg (1973). Os corpúsculos podem variar de tamanho e também em relação às espécies de animais. Por outro lado, as terminações nervosas de Meissner possuem axônios dispostos em diferentes níveis do corpúsculo (Cauna 1953, 1956, 1958, Ide 1975, 1976, 1977, Castano \& Ventura 1978, Watanabe \& Yamada 1988).

Estudos ultra-estruturais foram realizados da terminação nervosa da mucosa gengival de humanos (Martinez \& Pekarthy 1974, Watanabe \& Yamada 1983). Halata et al. (1999) relataram à presença dessas terminações nervosas na mucosa do palato mole de cabra, Watanabe \& Ide (1987) na mucosa do lábio de rato e Watanabe $\&$ Yamada (1985) na mucosa da bochecha de ratos. 
Estruturalmente, o corpúsculo de Herbst apresenta um axônio terminal e lamelas dispostas de forma concêntricas como tem sido relatados por Andres (1969), Nafstad \& Andersen (1970), Saxod $(1968,1970,1978)$ e Halata \& Munder (1980). Desta forma este trabalho teve por objetivo descrever os aspectos morfológicos dos componentes celulares, $\mathrm{o}$ axônio terminal e a cápsula dos corpúsculos de Herbst encontrados na mucosa palatina de avestruz, empregando os métodos de microscopia de luz.

\section{MATERIAL E MÉTODOS}

Mucosas palatinas de três avestruzes adultos foram divididas em partes anterior, médio e posterior e fixadas durante 24 horas em solução de formol a $10 \%$. Em seguida, os tecidos foram desidratados em série crescente de álcoois e incluídos em Paraplast. Cortes de $7 \mu \mathrm{m}$ foram montados em lâminas e corados com Hematoxilina-Eosina (HE), Tricômio de Masson e Picrosirius. Os cortes foram examinados e capturados em microscópio Zeiss com uma câmera digital acoplada (Axio Cam HRc-Zeiss ${ }^{\circledR}$ ) e os de Picrosirius foram observados com luz polarizada para a identificação dos tipos de fibras colágenas. Das imagens obtidas foi realizada a mensuração da área dos corpúsculos $\left(\mathrm{mm}^{2}\right)$, utilizando o programa de análise de imagem computadorizado Image-Pro-Plus 3.0.1 (Media Cybernetics, Silver Spring, Maryland, EUA).

\section{RESULTADOS}

Observou-se que os corpúsculos de Herbst estão localizados na lâmina própria do terço posterior da mucosa palatina de
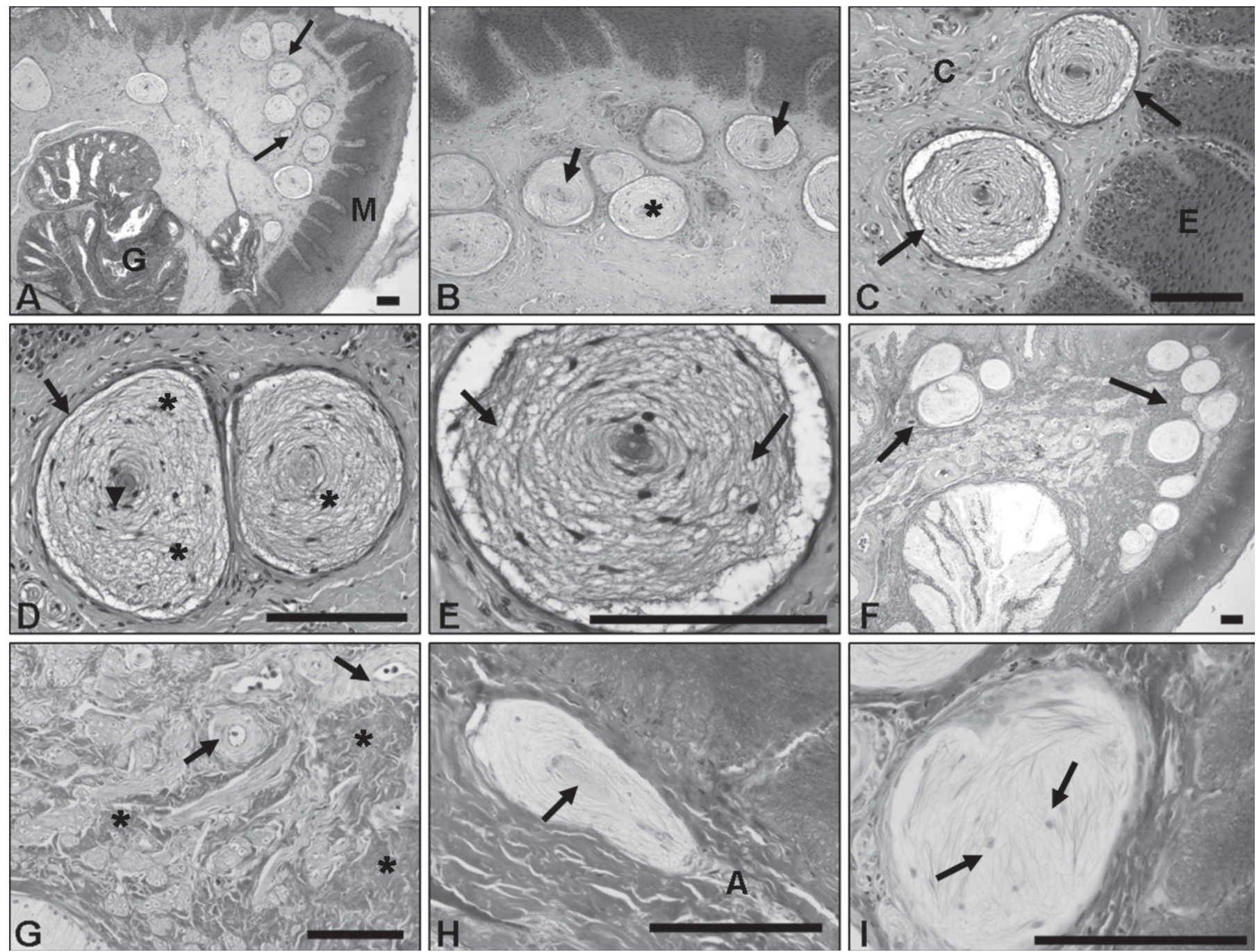

Fig.1. A. Corte frontal da mucosa palatina de avestruz, mostrando os corpúsculos subepiteliais de Herbst (setas), mucosa palatina (M) e glândulas salivares palatinas $(\mathrm{G})$. HE, Barra $100 \mu \mathrm{m}$. B. Cortes transversais de corpúsculos de Herbst, identificando-se o núcleo interno (seta), axônio terminal (*). HE, Barra $100 \mu \mathrm{m}$. C. Em maior aumento notam-se dois corpúsculos de Herbst (setas) localizados na porção subepitelial. (E) camada epitelial, (C) tecido conjuntivo. HE, Barra $100 \mu \mathrm{m}$. D. Os núcleos interno (cabeça de seta) e externo (") e a cápsula (setas) dos corpúsculos de Herbst podem ser identificados. HE, Barra $100 \mu \mathrm{m}$. E. Maior aumento mostra a distribuição dos feixes de fibras do núcleo externo (setas). HE, Barra $100 \mu \mathrm{m}$. F. Os corpúsculos de Herbst localizados no tecido conjuntivo junto à camada epitelial (setas). Tricômio de Masson, Barra $100 \mu \mathrm{m}$; G. Os feixes de fibras nervosas (setas) e tecido conjuntivo ("). Tricômio de Masson, Barra 100 $\mu \mathrm{m}$. H. Corte longitudinal do corpúsculo de Herbst, evidenciando o núcleo interno (seta maior) e fibras nervosas aferentes (A). Tricômio de Masson, Barra $100 \mu \mathrm{m}$. I. A rede de fibras da porção periférica e núcleos de células (setas). Tricômio de Masson, Barra $100 \mu \mathrm{m}$. 
avestruz, podendo ser encontrados agrupados ou isolados (Fig.1A). Estes distribuem-se regularmente na lâmina própria da mucosa palatina apresentando forma ovalada ou circular e rodeados de espessos feixes de fibras colágenas, com tamanho variando entre $1.238,6$ a $8.962,5 \mu \mathrm{m}^{2}$. Os corpúsculos podem estar separados ou fusionados no interior do tecido conjuntivo subepitelial (Fig.1C) da mucosa palatina. Os corpúsculos se constituem de uma cápsula externa, núcleos interno e externo e um axônio central (Fig.1B,D). Os núcleos das células lamelares são geralmente notados no pólo oposto do núcleo interno e lateralmente a eles (Fig.1E). A fibra nervosa amielínica penetra através da cápsula para o interior dos núcleos interno e externo apresentando um bulbo na porção terminal. Na coloração de Masson notam-se nitidamente fibras colágenas densas ao redor do corpúsculo de Herbst (Fig.1F). Próximo ao corpúsculo pode-se notar numerosos feixes de fibras nervosas mergulhados no interior do tecido conjuntivo denso (Fig.1G). Por outro lado, o núcleo interno é mais compacto e homogêneo em cortes longitudinais (Fig.1H). O núcleo externo é formado por várias camadas concêntricas de feixes de fibras colágenas entremeados de núcleos de fibroblastos e fibras delgadas (Fig.1I).

Notou-se a presença de uma rede contínua de lamelas que se prolonga através da cápsula externa, sendo os espaços interlamelares preenchidos por lâminas planas de fibroblastos (Fig.1I). O axônio terminal estende-se ao centro do corpúscu- lo, que é delimitado por uma série de células planas do tecido conjuntivo fibroso, as quais possuem um arranjo paralelo e provavelmente correspondem às células perineurais.

Quando se observa o conjunto de corpúsculos (Fig.2A) corado em Tricômio de Masson revela-se o tecido conjuntivo, corado em azul, delimitando os corpúsculos. Na coloração de Picrosirius revelou-se que o tecido conjuntivo adjacente estava nitidamente delimitado (Fig.2B) e quando examinado a luz polarizada este mostrou feixes de fibras semelhantes ao colágeno tipo I (Fig.2C). As Figuras 2D e 2E demonstram a distribuição de fibras do núcleo externo do corpúsculo de Herbst e sob luz polarizada observaram-se poucas fibras coradas com coloração esverdeada (Fig.2F).

\section{DISCUSSÃO}

O presente trabalho revela claramente a presença de terminações nervosas sensitivas lamelares no terço posterior da mucosa do palato de avestruz. Os corpúsculos de Herbst são notados no interior do tecido conjuntivo da lâmina própria e localizam-se em grupos ou isolados. Existem corpúsculos localizados bem próximos à lâmina basal das células epiteliais e outros localizados profundamente no interior dos ácinos das glândulas salivares.

Além disso, observou-se que os corpúsculos de Herbst são envoltos por um grande número de camadas concêntricas de extensões citoplasmáticas das células lamelares do núcleo
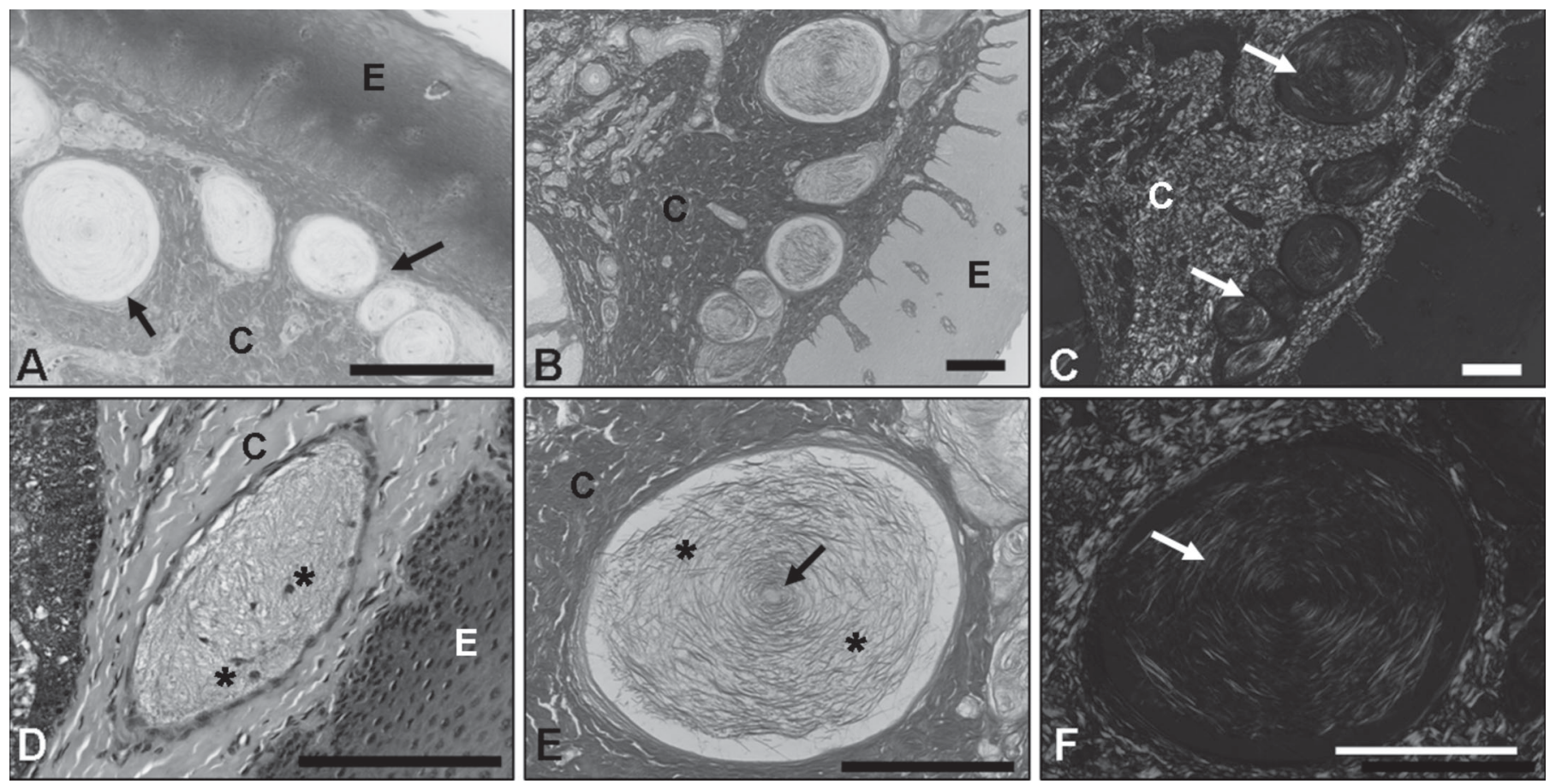

Fig.2. A. Presença de tecido conjuntivo (C) corado em azul, corpúsculos de Herbst (setas) e camada epitelial (E). Tricômio de Masson, Barra $100 \mu \mathrm{m}$. B. Em aspecto vermelho, o tecido conjuntivo (C) e camada epitelial (E). Picro-sírius, Barra 100 $\mu \mathrm{m}$. C. Região anterior examinada sob luz polarizada, mostrando a presença de fibras de aspecto esverdeado assemelhando a fibras colágenas tipo I (setas) e fibras colágenas subepiteliais (C). Picrosirius, 200x, Barra 100 $\mu \mathrm{m}$. D. Disposição de fibras do núcleo externo do corpúsculo de Herbst ("), camada epitelial (E) e tecido conjuntivo subepitelial (C). HE, Barra $100 \mu \mathrm{m}$. E. Disposição das fibras do núcleo externo do corpúsculo de Herbst ("), axônio central (seta) e tecido conjuntivo periférico (C). Picro-sírius, Barra $100 \mu \mathrm{m}$. F. Luz polarizada revela a presença de poucas fibras do tipo I (setas). Picrosirius, Barra $100 \mu \mathrm{m}$. 
interno e externo. As lamelas da cápsula externa e o núcleo interno são evidenciados em cortes histológicos corados em Hematoxilina-Eosina e Masson. Os nossos resultados revelaram que as características dos corpúsculos de Herbst são semelhantes àqueles relatados anteriormente por Saxod (1968, 1970, 1978) e Watanabe et al. (1985). Salienta-se que o núcleo interno apresentando a terminação nervosa sensitiva, o externo com a série de lamelas concêntricas e à cápsula externa ocupada pelo prolongamento citoplasmático das células planas em associação com as fibras colágenas foram identificados por Andersen \& Nafstad (1968) no palato de galinhas, e por Nafstad \& Andersen (1970) em corpúsculos de Herbst de pintinhos.

As lamelas externas são maiores e compostas por numerosas linhas concêntricas e espaços interlamelares amplos, contendo substâncias amorfas, assemelhando-se com os dados relatados por Watanabe et al. (1985). Entretanto, os corpúsculos do bico de pintinhos (Nafstad \& Andersen 1970) apresentam uma forma oval alongada e tem dimensões variáveis. O núcleo interno do corpúsculo de pintinhos é composto por cerca de 50 lâminas citoplasmáticas (Saxod 1968, 1978) e a cápsula externa possui menor quantidade de lamelas do que encontrada neste trabalho. $\mathrm{O}$ núcleo interno do corpúsculo de Herbst relatado por Watanabe et al. (1985) revela uma organização muito simétrica, contendo em cada lado de 60 a 70 lâminas citoplasmáticas, medindo cerca de 0,1 micrômetro de largura. Estas características são relatadas também por Andres (1969), Saxod (1968, 1970, 1978), Halata \& Munger (1980), Polacek et al. (1966) e Malinovsky \& Zemanek (1970) ao quais descrevem corpúsculos de Herbst na membrana da cápsula articular de pássaros. Saxod $(1968,1970)$ relatou que existem dois tipos de corpúsculos de Herbst na cápsula articular de pombos. Pode-se afirmar que, além dessas características, o corpúsculo de Herbst acha-se envolto por 10-15 lâminas de células planas, dispostas paralelamente, as quais correspondem às células prineurais dos nervos periféricos, conforme foi descrito por Shanthaveerappa \& Bourne (1963), Santini (1969) e por Munger (1971). Os corpúsculos de Herbst são considerados mecanorreceptores de rápida adaptação importantes na transmissão dos impulsos táteis e pressão da mucosa oral em aves.

\section{REFERÊNCIAS}

Andersen A.E. \& Nafstad P.H.J. 1968. An electron microscopic investigation of the sensory organs in the hard palate region of the hen. Z. Zellforch. 91:281-401.

Andres K.H. 1969. Zur Ultrastruktur verschiedener Mechanorezeptoren von höheren Wirbeltieren. Anat. Anz. 124:551-565.

Castano P. \& Ventura R.G. 1978. The Meissner corpuscle of the green-monkey: The organization of the nervous component. J. Submicrosc. Cytol. 10:327344.

Cauna N. 1953. Some observations on the structure and development of Meissner's corpuscle. J. Anat. 87:440-441.

Cauna N. 1956. Structure ande origin of the capsule of Meissner's corpuscle. Anat. Rec. 124:77-93.
Cauna N. 1958. Structure of digital touch corpuscles. Acta Anat. 32:1-23.

Cauna N. \& Mannan G. 1958. Development and postnatall changes of digital pacinian corpuscles in the human hand. J. Anat. 92:1-20.

Halata Z. \& Munger B.L. 1980. The ultrastructure of Ruffini and Herbst corpuscles in the articular capsule of domestic pigeon. Anat. Rec. 198:681-692.

Halata Z., Cooper B.Y., Baumann K.I., Schwegmann C. \& Friedman R.M. 1999. Sensory nerve endings in the hard palate and papilla incisiva of the goat. Exp. Brain Res. 129:218-28.

Idé C. \& Munger B.L. 1978. A cytologic study of Grandry corpuscle development in chicken toe skin. J. Comp. Neurol. 179:301-324.

Idé C. 1975. Morphological studies on the development of the Meissnertype corpuscle of the mouse. Proc.10th Congr. Anat., Tóquio, Japão. 305p.

Idé C. 1976. The fine structure of the digital corpuscle of the mouse toe pad, with special reference to nerve fibers. Am. J. Anat. 147:329-356.

Idé C. 1977. Development of Meissner corpuscle of mouse toe pad. Anat. Rec. 188:49-68.

Malinovsky L. \& Zemanek R. 1970. Sensory nerve endings in the joint capsules of the large limb joints in the domestic hen and the rook. Folia Morphol. 18:206-212.

Martinez Z.R. \& Pekarthy J.M. 1974. Ultrastructure of encapsulated nerve endings in rat gingiva. Am. J. Anat. 140:135-138.

Munger B.L. 1971. Patterns of organization of peripheral sensory receptors, p.523-556. In: Leowenstein W.R. (ed.), Handbook of Sensory Physiology. Springer-Verlag, Berlin.

Nafstad P.H.J. \& Andersen A.E. 1970. Ultrastructural investigation on the innervation of the Herbst corpuscle. Z. Zellforch. 103:109-114.

Polacek P.C., Skleusk A. \& Malinovsky L. 1966. Contribution to the problem of joint receptors in birds. Folia Morphol. 14:33-42.

Pease D.C. \& Quilliam T.A. 1957. Electron microscopy of the Pacinian corpuscle. J. Biophys. Biochem. Cytol. 3:331-357.

Saxod R. 1968. Ultrastructure des corpuscles sensorieris cutánés de Herbst et de Grandry chez le canard. Arch. Anat. Micr. Morphol. Exp. 57:379-400.

Saxod R. 1970. Etude au microscope eletronique de l'histogenese du corpuscle sensorial cutané de Herbst chez le Canard. J. Ultra. Res. 33:463-482.

Saxod R. 1978. Development of cutaneous sensory receptors in birds, p.339417. In: Jacobson M. (ed.), Handbook of Sensory Physiology: development of sensory systems. Vol.9. Springer-Verlag, New York.

Spencer P.S. \& Schaumburg H.H. 1973. An ultrastructural study of the inner core the Pacinian corpuscle. J. Neurocy. 2:217-235.

Shanthaveerapa T.R. \& Bourne G.H. 1963. New observations on the structure of the Pacinian corpuscle and its relation to the perineural epithelium of peripheral nerves. Am. J. Anat. 112:97-109.

Santini M. 1969. Numbers of Pacinian corpuscles of the cat pancreas, mesentery and mesocolon. Anat. Rec. 113:322A.

Watanabe I. \& Yamada E. 1983. The fine structure of lamellated nerve endings found in the rat gingiva. Arch. Hist. Jap. 46:173-182.

Watanabe I. \& Yamada E. 1985. Light and electron microscopy study of lamellated nerve endings found in the rat cheek mucosa. Arch. Hist. Jap. 48:497-504.

Watanabe I., Usukura J. \& Yamada E. 1985. Electron microscopy study of the Grandry and Herbst corpuscles found in the palatine mucosa, gingival mucosa and beak skin of the duck. Arch. Hist. Jap. 48:1-9.

Watanabe I. \& Ide C. 1987. Cholinesterase activity of lamellated sensory corpuscles in rat lip. Arch. Oral Biol. 32:129-134.

Watanabe I. \& Yamada E. 1988. Fine structure of the lamellated corpuscles in the mouse hard palatine mucosa. Z. Mikrosk. Anat. Forsch. 102:811-816.

Watanabe I. \& Yamada E. 1989. Fine structure of lamellated and free nerve endings of the rat soft palate mucosa. Revta Microsc. Eletr. Biol. Celular 13:139-150. 